\title{
A phase I dose escalation study of S-1 with concurrent radiotherapy in elderly patients with esophageal cancer
}

\author{
Yongling $\mathrm{Ji}^{1,2}$, Guoqing Qiu ${ }^{1,2}$, Liming Sheng ${ }^{1,3}$, Xiaojiang Sun ${ }^{1,3}$, Yuanda Zheng ${ }^{1,2}$, Ming Chen ${ }^{1,2}$, Xianghui Du ${ }^{1,2}$ \\ ${ }^{1}$ Department of Radiation Oncology, Zhejiang Cancer Hospital, Hangzhou 310022, China; ${ }^{2}$ Key Laboratory of Radiation Oncology, Hangzhou \\ 310022, China; ${ }^{3}$ Key Laboratory of Diagnosis and Treatment Technology on Thoracic Oncology, Hangzhou 310022, China \\ Contributions: (I) Conception and design: Y Ji, X Du; (II) Administrative support: M Chen; (III) Provision of study materials or patients: Y Ji, M Chen, \\ X Du; (IV) Collection and assembly of data: L Sheng, X Sun; (V) Data analysis and interpretation: Y Ji, G Qiu, L Sheng, X Du; (VI) Manuscript \\ writing: All authors; (VII) Final approval of manuscript: All authors. \\ Correspondence to: Xianghui Du. Department of Radiation Oncology, Zhejiang Cancer Hospital, 38 Guangji Road, Hangzhou 310022, China. \\ Email: jiyl@zjcc.org.cn.
}

\begin{abstract}
Background: Concurrent chemoradiotherapy (CRT) with 5-fluorouracil (5-FU) and cisplatin (CDDP) are often associated with significant incidence of toxic effects in elderly patients with esophageal cancer. This phase I trial was designed to determine the maximum tolerated dose (MTD) and dose-limiting toxicity (DLT) of S-1, an oral 5-FU derivative, when given with radiotherapy in elderly patients.

Methods: Patients who were age of 70 years or older with histologically confirmed esophageal cancer, and had an Eastern Cooperative Oncology Group (ECOG) score of 0-2 were eligible for this study. Radiotherapy was administered in 1.8 Gy fractions 5 times weekly to a total dose of 54 Gy. S-1 was administered on days 1-14 and 29-42 at the following dosages: 60, 70, and $80 \mathrm{mg} / \mathrm{m}^{2} /$ day. Trial registration: NCT01175447 (ClinicalTrials.gov).

Results: Twelve previously untreated patients were enrolled in this study. No grade 3 or 4 toxicity was observed in six patients treated at the 60 and $70 \mathrm{mg} / \mathrm{m}^{2}$ dose levels. DLT was observed in four of six patients treated at the $80 \mathrm{mg} / \mathrm{m}^{2}$ dose level. Two patients developed grade 3 esophagitis, one patient developed grade 3 esophagitis and pneumonitis, and one patient developed grade 3 thrombocytopaenia. Endoscopic complete response (CR) was observed in eight patients $(66.7 \%)$. The median progression free survival (PFS) was 20 months and median overall survival was 29 months.

Conclusions: The MTD of S-1 was $80 \mathrm{mg} / \mathrm{m}^{2}$, and the recommended dose (RD) for phase II studies was $70 \mathrm{mg} / \mathrm{m}^{2}$. This regimen was well tolerated and active in elderly patients with esophageal cancer, meriting further investigation in phase II studies.
\end{abstract}

Keywords: S-1; chemoradiotherapy (CRT); esophageal cancer; elderly

Submitted Feb 04, 2015. Accepted for publication Nov 16, 2015.

doi: $10.21037 /$ jtd.2016.02.70

View this article at: http://dx.doi.org/10.21037/jtd.2016.02.70

\section{Introduction}

Esophageal cancer is the eighth most common cancer and the sixth cause of cancer mortality worldwide (1). In the United States, $44 \%$ and $30 \%$ esophageal cancer occurs in patients over 60 and 75 years of age respectively (2). Similar situation was observed in China, the region with one of the highest incidences of esophageal cancer in the world (3). Approximately $30-40 \%$ of the total esophageal cancer patients were 70 years old or above (4). Because China is rapidly becoming an aging society, the number of elderly patients with esophageal cancer is likely to increase significantly in the near future.

Surgery is the most important approach for radical treatment of esophageal cancer. However, elderly patients were more likely to be referred to non-surgical treatment, 
partially because of medical comorbidities and reduced functional reserve of organs (5-7). A population-based study of the National Cancer Registry in Ireland showed that, when compared with patients younger than 60 years of age, the likelihood for resection was significantly lower among older cohorts by $33 \%, 74 \%$ and $93 \%$ for patients aged 60-69, 70-79 and 80+, respectively (7).

Chemoradiotherapy (CRT) has been accepted nowadays as the standard nonsurgical treatment for locally advanced esophageal cancer. The Radiation Therapy Oncology Group (RTOG) phase III intergroup trial RTOG 85-01 demonstrated that CRT with 5-fluorouracil (5-FU) and cisplatin (CDDP) provided a significant survival advantage over RT alone $(8,9)$. However, the toxicity of CRT was substantial. $64 \%$ of patients treated with CRT experienced severe or life threatening adverse events comparing to $28 \%$ of patients treated with RT alone (9). And only $23 \%$ of patients enrolled in this study were over age 70, which brought a question about the suitability of CRT for elderly patients. Recently, several retrospective studies suggested that elderly patients with esophageal cancer may also benefit from CRT with 5-FU and CDDP (10-12). The clinical complete response (CR) rate was found at a range of 57.8-63.6\% and survival time of 8.6-15.2 months. However, only $9-38.5 \%$ of patients finished the scheduled treatment because of the significant incidence of toxic effects (10-12). Moreover, a study on patients aged over 75 demonstrated a treatment-related mortality rate of up to $18 \%$ (13). Therefore, potent new CRT regimens with lower toxicity are needed to improve the treatment outcome for elderly patients.

S-1 is a novel agent designed to enhance anticancer activity and to reduce toxicity through the combined use of an oral fluoropyrimidine agent (tegafur), a dihydropyrimidine dehydrogenase inhibitor (5-chloro-2,4-dihydroxypyridine), and an orotate phosphoribosyl transferase inhibitor (potassium oxonate) (14). S-1 has been shown to have one of the highest response levels as a single agent for metastatic stomach cancer, non-small cell lung cancer, and colorectal cancer with mild toxicity (15-19). S-1 could be expected to have several advantages over 5-FU in CRT. First, prolonged exposure is desirable in order to achieve radiosensitisation. Studies have shown that the half-life of 5-FU after oral S-1administration was markedly prolonged compared with that of 5-FU after intravenous administration (20,21). Second, gimeracil, a component of S-1, has been found to enhance the efficacy of RT through the inhibition of the repair of radiation-induced DNA damage $(22,23)$. A preclinical study using human cancer xenograft models showed that oral S-1 produced better response than intravenous 5-FU in the CRT (23). Moreover, S-1 is administered orally; it can be given on a daily basis, which is convenient for fractionated radiotherapy.

In the light of these data, we conducted a phase I trial to evaluate the maximum-tolerated dose (MTD), the recommended dose (RD), and the dose-limiting toxicity (DLT) of S-1 in combination with radiotherapy in elderly patients older than 70 years with esophageal cancer (ClinicalTrials.gov identifier NCT01175447).

\section{Materials and methods}

\section{Eligibility criteria}

Eligible patients were required to meet the following criteria: (I) histologically confirmed squamous cell carcinoma or adenocarcinoma; (II) stage I to IV diseases according to the 2002 (version 6.0) American Joint Committee on Cancer staging system, with the exception of stage $\mathrm{IVb}$ of distant and hematogenous visceral metastasis (eligible if it was lymph node metastases); (III) Eastern Cooperative Oncology Group (ECOG) performance status (PS) score of 0-2; (IV) age $\geq 70$ years old; (V) 12 weeks or more life expectancy; (VI) adequate bone marrow reserve (leukocyte count $\geq 4,000 \mathrm{~mm}^{3}$, neutrophil count $\geq 2,000 \mathrm{~mm}^{3}$, platelet count $\geq 100,000 \mathrm{~mm}^{3}$, and haemoglobin $\geq 10 \mathrm{~g} / \mathrm{dL}$ ); (VII) normal liver function (total serum bilirubin $\leq 1.5 \mathrm{mg} / \mathrm{dL}$, with aspartate transaminase and alanine transaminase levels being lower than double of the upper normal limit); (VIII) normal renal function (normal serum creatinine and blood urea nitrogen levels); and (IX) adequate pulmonary function $\left(\mathrm{FEV}_{1}>1 \mathrm{~L}\right)$. Patients were excluded if they had one of the following: (I) malignant pleural or pericardial effusion; (II) a concomitant serious illness such as uncontrolled angina pectoris; (III) myocardial infarction in previous 3 months; (IV) heart failure; (V) interstitial pneumonia; or (VI) infection or other diseases contraindicating chemotherapy or RT. The study was approved by the Ethics Committee of Zhejiang Cancer Hospital, and the written informed consent was obtained from all patients.

\section{Pre-treatment evaluation}

The pre-treatment evaluation included history, physical examination, electrocardiography, and assessment of bone marrow, renal, and hepatic functions. The items of the 
disease evaluation included a neck, chest and abdominal CT, an upper GI endoscopy, endoscopic ultrasound (EUS), and barium oesophagraphy. Bronchoscopy was performed for cervical or midesophageal tumours. A brain MRI, and radionuclide bone scan were performed if clinically indicated. PET-CT scan was recommended but not a requisite part of the pre-treatment evaluation. The clinical TNM system stage was determined according to the 2002 (version 6.0) American Joint Committee on Cancer staging system. The Charlson score was used for the analysis of patient comorbidity (24).

\section{Treatment}

Patients received an oral dose of S-1 twice daily after meal on days 1-14 and 29-42. A powder form of S-1 would be administered if patients could not swallow the oral capsule. The starting S-1 dose was $60 \mathrm{mg} / \mathrm{m}^{2} /$ day, which represents $75 \%$ of the daily monotherapeutic dose. The doses were escalated as follows: 60,70 , and $80 \mathrm{mg} / \mathrm{m}^{2} /$ day, and no dose modification was permitted at each dose level. Because S-1 is only available for use in 20- or 25-mg capsules, the individual dose was rounded down to the nearest pill size less than the calculated dose. Antiemetic drugs were not routinely used.

RT was administered beginning on day 1 of chemotherapy using a linear accelerator $(10 \mathrm{MeV})$. Each patient received a single 1.8 Gy daily fraction for 5 consecutive days each week, until a total dose of 54 Gy was reached. Each patient underwent a treatment-planning CT scan with intravenous contrast. The gross tumour volume (GTV) was contoured based on the EUS, barium oesophagraphy and chest CT scans. The clinical target volume (CTV) consisted of the GTV plus a $0.5-1 \mathrm{~cm}$ circumferential margin and $3-4 \mathrm{~cm}$ cranio-caudal margin. The supraclavicular nodes were included for upper esophageal lesions, and celiac nodes were included for distal esophageal lesions. The planning target volume (PTV) consisted of the CTV plus a $0.5-1 \mathrm{~cm}$ margin for daily set-up error and organ motion. Dose-volume histogram analysis was required to ensure that the spinal cord, lung, heart, and liver exposure were within organ tolerance. There are no consolidate chemotherapy after concurrent CRT in our study.

\section{Assessment of toxicity and dose modifications}

Toxicity was assessed based on the National Cancer Institute Common Terminology Criteria for Adverse Events v3.0. A complete blood cell count and serum chemistry profile was performed at least once a week during treatment. Non-hematologic toxicity was evaluated on a daily basis via interview and physical examination throughout the treatment period. If grade 3 neutropenia alone occurred, S-1 was held and RT continued. S-1 was then restarted at the same dose when the absolute neutrophil count became $\geq 1,000 / \mathrm{mm}^{3}$. If other grade 3 or 4 toxicities occurred, both RT and S-1 were held until recovery to grade 2 .

\section{Dose escalation}

DLT was defined as the development of at least one of the following adverse events: grade 4 hematologic toxicity; fever (i.e., body temperature $\geq 38^{\circ} \mathrm{C}$ ) with grade 3 neutropenia; grade 3 or higher thrombocytopenia; any RT delay for 2 weeks or more; any delay of the scheduled oral intake of S-1 for 2 weeks or more; and grade 3 or higher nonhematologic toxicity. Initially, three patients were scheduled to enter the study at each dose level; if all three patients at a given dose level developed DLT, that dose was designated as the maximum tolerated dose (MTD). If only two patients experienced DLT, three additional patients were subjected to the same dose level. The MTD was defined as the dose at which three or more out of six patients developed any of the specified DLTs. The dose level immediately below the MTD was considered the RD for phase II studies.

\section{Response assessment}

Clinical response was assessed according to Response Evaluation Criteria in Solid Tumours (RECIST) (25). The primary tumour was assessed on CT scan with the measures of the vertical length and maximal thickness of the esophageal wall on transverse plane. Endoscopic CR was assessed according to the criteria proposed by Tahara et al., which was defined upon observation of the entire esophagus as: (i) disappearance of the tumour lesion; (ii) disappearance of ulceration (slough); and (iii) absence of cancer cells in biopsy specimens (26). This evaluation was performed 5-6 weeks after CRT completion. Endoscopy and CT scans were performed every 3 months during follow-up.

\section{Results}

\section{Patient characteristics}

Between February 2010 and December 2011, 12 patients with a median age of 74 years (range, 70-83 years) were 
Table 1 Patient characteristics

\begin{tabular}{|c|c|c|}
\hline Characteristic & $\begin{array}{c}\text { No. of } \\
\text { patients }(n=12)\end{array}$ & $\begin{array}{c}\text { Valid } \\
\text { patients (\%) }\end{array}$ \\
\hline \multicolumn{3}{|l|}{ Age (y) } \\
\hline Median (range) & \multicolumn{2}{|c|}{74 [70-83] } \\
\hline \multicolumn{3}{|l|}{ Sex } \\
\hline Male & 9 & 75.0 \\
\hline Female & 3 & 25.0 \\
\hline \multicolumn{3}{|l|}{ Histology } \\
\hline Squamous cell carcinoma & 12 & 100.0 \\
\hline Adenocarcinoma & 0 & 0 \\
\hline \multicolumn{3}{|l|}{ ECOG performance status } \\
\hline \multicolumn{3}{|l|}{ score } \\
\hline 0 & 1 & 8.3 \\
\hline 1 & 11 & 91.7 \\
\hline \multicolumn{3}{|l|}{ Tumour locations } \\
\hline Cervical & 1 & 8.3 \\
\hline Upper third & 5 & 41.7 \\
\hline Middle third & 4 & 33.3 \\
\hline Lower third & 2 & 16.7 \\
\hline \multicolumn{3}{|l|}{ Stage } \\
\hline I & 1 & 8.3 \\
\hline Ila & 2 & 16.7 \\
\hline $\mathrm{Ilb}$ & 1 & 8.3 \\
\hline III & 5 & 41.7 \\
\hline IVa & 1 & 8.3 \\
\hline $\mathrm{IVb}$ & 2 & 16.7 \\
\hline \multicolumn{3}{|l|}{ Tumour length } \\
\hline$<5 \mathrm{~cm}$ & 4 & 33.3 \\
\hline$\geq 5-<10 \mathrm{~cm}$ & 6 & 60.0 \\
\hline$\geq 10 \mathrm{~cm}$ & 2 & 16.7 \\
\hline \multicolumn{3}{|l|}{ Histologic grade ${ }^{a}$} \\
\hline $\mathrm{Gx}$ & 2 & 16.7 \\
\hline G1 & 2 & 16.7 \\
\hline G2 & 6 & 50 \\
\hline G3 & 2 & 16.7 \\
\hline G4 & 0 & 0 \\
\hline \multicolumn{3}{|l|}{ Charlson score } \\
\hline Median (min-max) & \multicolumn{2}{|c|}{$1[0-3]$} \\
\hline Charlson score 2 or more & 4 & 33.3 \\
\hline
\end{tabular}

a, Gx, grade could not be assessed; G1, well differentiated; G2, moderately differentiated; G3, poorly differentiated; G4, undifferentiated.
Table 2 Principal toxicities

\begin{tabular}{lccc}
\hline Toxicity & $\begin{array}{c}\text { Dose level 1 } \\
(\mathrm{n}=3) 1 / 2 / 3 / 4\end{array}$ & $\begin{array}{c}\text { Dose level } 2 \\
(\mathrm{n}=3) 1 / 2 / 3 / 4\end{array}$ & $\begin{array}{c}\text { Dose level } 3 \\
(\mathrm{n}=6) 1 / 2 / 3 / 4\end{array}$ \\
\hline Anaemia & $1 / 1 / 0 / 0$ & $3 / 0 / 0 / 0$ & $2 / 3 / 0 / 0$ \\
Leukopenia & $2 / 1 / 0 / 0$ & $2 / 1 / 0 / 0$ & $3 / 2 / 1 / 0$ \\
Neutropenia & $3 / 0 / 0 / 0$ & $2 / 1 / 0 / 0$ & $4 / 2 / 0 / 0$ \\
Thrombocytopenia & $0 / 0 / 0 / 0$ & $0 / 1 / 0 / 0$ & $1 / 1 / 1^{*} / 0$ \\
Esophagitis & $0 / 3 / 0 / 0$ & $0 / 3 / 0 / 0$ & $0 / 3 / 3^{*} / 0$ \\
Pneumonitis & $1 / 1 / 0 / 0$ & $0 / 1 / 0 / 0$ & $1 / 1 / 1^{\star} / 0$ \\
Nausea & $1 / 0 / 0 / 0$ & $2 / 1 / 0 / 0$ & $5 / 1 / 0 / 0$ \\
Diarrhoea & $0 / 0 / 0 / 0$ & $0 / 0 / 0 / 0$ & $0 / 1 / 0 / 0$ \\
Anorexia & $1 / 1 / 0 / 0$ & $2 / 1 / 0 / 0$ & $4 / 2 / 0 / 0$ \\
\hline
\end{tabular}

*, DLT, dose-limiting toxicity.

enrolled in this study. The patient clinical characteristics were detailed in Table 1. All patients had squamous cell carcinoma. There were eight stage III-IV patients (66.7\%), among whom two patients were at stage IVb (16.7\%), and both had distant lymph node metastasis but no visceral organ metastasis. In most patients $(8 / 12,66.7 \%)$, the tumour length was greater than $5 \mathrm{~cm}$. Nine cases $(75.0 \%)$ were associated with other diseases, including six cases of peripheral vascular disease $(50 \%)$, four cases of chronic obstructive pulmonary disease (33.3\%), and one case each of diabetes, heart disease, ulcers, and cerebrovascular disease (8.3\%). The median Charlson score was 1 (range, 0-3). Nine patients $(75.0 \%)$ had a Charlson score $\geq 1$, and four patients (33.3\%) had a Charlson score $\geq 2$.

\section{Toxicity}

All 12 patients received a toxicity evaluation. The results are shown in Table 2. DLT was not observed at level $1\left(60 \mathrm{mg} / \mathrm{m}^{2}\right)$ and level $2\left(70 \mathrm{mg} / \mathrm{m}^{2}\right)$. At level $3\left(80 \mathrm{mg} / \mathrm{m}^{2}\right.$ of S-1), two patients of the first three developed grade 3 esophagitis. The third patient developed grade 3 leucopoenia, resulting in delayed S-1 chemotherapy for 4 days. Because two of three patients developed DLT, an additional three patients were enrolled at level 3 . One patient developed grade 3 thrombocytopenia, resulting in delayed S-1 chemotherapy for more than 14 days. Another patient developed grade 3 esophagitis and grade 3 pneumonia. Both RT and chemotherapy were terminated, and the esophagitis and pneumonia were relieved by steroid and antibiotic 
treatment. One patient did not show any grade 3 or greater toxicity. Therefore, the dose level 3 was considered to be the MTD because four of the six patients presented with DLT, and dose level 2 was recommended as the dose for phase II trials. The incidences of grade 3 toxicity were $40.0 \%$ and $42.9 \%$ in patients with age $\geq 75$ and $<75$ years old, respectively.

\section{Treatment delivery}

The powder form of S-1 was administered in five patients who could not swallow the oral capsule. Among them, a feeding tube was inserted before CRT in two patients with severe esophageal stenosis. Full dose chemotherapy was completed without delay in seven patients (58.3\%). One patient restarted S-1 administration on day 39 instead of day 35 because of leucopoenia. Four patients could not take S-1 for 4, 6, 7, and 11 days because of esophagitis and pneumonitis, esophagitis, esophagitis, and thrombocytopenia, respectively. Full dose RT was completed in 11 patients $(91.7 \%)$, three of whom had RT interruption for 3, 4, and 7 days, respectively. One patient could not complete planned RT (total dose: $52.2 \mathrm{~Gy}$ ) because of esophagitis and pneumonitis. The mean lung dose in this patient was $12.38 \mathrm{~Gy}$, and the percent of the total lung volume exceeding 20 Gy (V20) was 26\%.

\section{Response and follow-up}

Responses to therapy and disease status during the follow-up were summarized in Table 3. Endoscopic CR was observed in eight patients (66.7\%). According to RECIST criteria, two $(16.7 \%)$ patients achieved CR, nine (75\%) patients achieved partial response (PR), and one (8.3\%) patient had stable disease.

At a median follow-up of 29 months (range, 6-36 months), two patients (16.7\%) developed local progression, and four patients developed distant metastases. Five patients were still alive (41.7\%), all of whom had endoscopic CR. Five patients died of tumour progression. One patient with T4N1M0 diseases (tumor invades trachea) died of esophageal hemorrhage 8 months after the completion of protocol treatment. There was no evidence of tumor progression, aortic invasion, deep esophageal ulcer and tracheoesophageal fistula from chest CT and endoscopy during follow-up. The massive hemorrhage perhaps caused by tumor necrosis. However, the exact reason was unknown because the patient was died at home and no autopsy was done. One patient who had no evidence of neoplasm at the time of death died of cerebral infarction 5 months after enrolling in this trial. The median survival time and median progression free survival (PFS) time were 29 and 20 months, respectively.

\section{Discussion}

This is the first report of a phase I study of CRT using S-1 in the treatment of esophageal cancer in elderly patients. Esophagitis was the most common toxicity in our study, with grade 3 esophagitis observed in 3 of 12 patients. Haematological toxicity was mild, with grade 3 thrombocytopenia and leukopenia observed in one and one patients, respectively. Grade 3 pneumonia was observed in one patient. No grade 4 toxicity or treatment-related deaths was observed. The endoscopic CR rate was $66.7 \%$; the median survival time was 29 months. Compared with conventional CRT using 5-FU and CDDP in elderly patients (10-12), our regimen seems to be well tolerated and active, although the efficacy was not a primary object in this small phase I study.

Previously, several studies suggested that the elderly patients with esophageal cancer could benefit from CRT with 5-FU and CDDP, but the treatment compliance was quite low due to adverse events $(10,11,27)$. Tougeron et al. (10) reported a study with 109 patients older than 70 years receiving CRT with 5-FU/CDDP (n=98) or CPT-11/CDDP (n=10). Only $38.5 \%$ of patients completed the planned treatment. The CR rate was $57.8 \%$; the median survival was 15.2 months. Takeuchi et al retrospectively analyzed the results of CRT with 5-FU and CDDP in 33 elderly patients and 145 non-elderly patients. They found that the elderly patients demonstrated much lower treatment compliance than non-elderly patients, with only $33.3 \%$ of the elderly completed the planned treatment, comparing to $68.3 \%$ of non-elderly patients. Toxicity caused by CRT to elderly patients was significantly higher than that to non-elderly patients. The incidences of over grade 3 leukopenia was $70.0 \%$ in elderly patients versus $49.7 \%$ in non-elderly, anaemia at $51.5 \%$ versus $17.9 \%$, and thrombocytopenia at $33.3 \%$ versus $18 \%$ (11).

As it is difficult to reach a standard dose intensity for a two-drug combination regimen with concurrent radiotherapy in elderly patients with esophageal cancer, CRT with single-agent chemotherapy may be a reasonable alternative. Servagi-Vernat et al. (28) reported 22 patients with stage II-III esophageal cancer over the age of 75 received a 50 Gy RT concurrent with CDDP $75 \mathrm{mg} / \mathrm{m}^{2}$ on days 1 and 21. All 
Table 3 Response and survival

\begin{tabular}{|c|c|c|c|c|c|c|c|c|c|c|}
\hline Patient No & & Age & $\begin{array}{l}\text { Tumour } \\
\text { locations }\end{array}$ & TNM stage & $\begin{array}{c}\text { Clinical } \\
\text { response- } \\
\text { RECIST criteria }\end{array}$ & $\begin{array}{c}\text { Endoscopic } \\
\text { CR }\end{array}$ & $\begin{array}{l}\text { Site of } \\
\text { recurrence }\end{array}$ & $\begin{array}{c}\text { PFS } \\
\text { (months) }\end{array}$ & $\begin{array}{l}\text { Survival } \\
\text { time } \\
\text { (months) }\end{array}$ & Follow-up \\
\hline Cohort 1 & 3 & 77 & Middle third & T4N1M1b & SD & No & Liver, brain & 5 & 11 & $\begin{array}{l}\text { Died of tumour } \\
\text { progression }\end{array}$ \\
\hline \multirow[t]{2}{*}{ Cohort 2} & 4 & 71 & Upper third & T2N1M1a & PR & Yes & Lung & 7 & 12 & $\begin{array}{l}\text { Died of tumour } \\
\text { progression }\end{array}$ \\
\hline & 6 & 74 & Upper third & T2NOMO & $\mathrm{CR}$ & Yes & None & 38 & 38 & Alive \\
\hline \multirow[t]{5}{*}{ Cohort 3} & 7 & 70 & Upper third & T1N0M0 & $\mathrm{CR}$ & Yes & None & 36 & 36 & Alive \\
\hline & 8 & 70 & Lower third & T2NOMO & PR & Yes & Local, liver & 16 & 29 & $\begin{array}{l}\text { Died of tumour } \\
\text { progression }\end{array}$ \\
\hline & 9 & 77 & Cervical & T4N1M0 & PR & Yes & None & 34 & 34 & Alive \\
\hline & 10 & 72 & Upper third & T2N1M0 & PR & Yes & None & 32 & 32 & Alive \\
\hline & 11 & 73 & Upper third & T4N1M0 & PR & No & Local & 5 & 9 & $\begin{array}{l}\text { Died of tumour } \\
\text { progression }\end{array}$ \\
\hline
\end{tabular}

RECIST, Response Evaluation Criteria in Solid Tumours; CR, complete response; PFS, progression free survival; PR, partial response; $\mathrm{SD}$, stable disease.

patients completed the planned treatment, with no grade 4 toxicity observed. A total of $63.6 \%$ patients achieved CR, $36.4 \%$ of patients experienced no tumour effect or local progression, and the median survival time was 15 months. In our study, all patients at the RD or lower dose level completed the planned CRT, only one patient at the MTD could not complete planned RT (total dose: 52.2 Gy). The endoscopic CR rate was $66.7 \%$, and only two patients (16.7\%) developed local progression at a median follow-up of 29 months. However, because of the small number of patients in these studies, further trials will be necessary to evaluate the role of CRT with single-agent chemotherapy in elderly patients.

Recently, CRT with S-1 and platinum for nonage-selected patients with esophageal cancer had been investigated. Iwase et al. (29) reported a phase II study of 116 patients receiving CRT with S-1 and CDDP. The median survival time was $7.0,2.6$, and 1.3 years for the stage II, III, and IVa patients, respectively. The most serious toxicity was myelosuppression: grade 3 and 4 neutropenia occurred in $28.4 \%$ and $9.5 \%$ of patients, respectively. In a prospective study of CRT with S-1 and nedaplatin in 20 patients with stage II/III esophageal cancer, the 3 -year overall survival rate was $58.0 \%$. The reported toxicities were grade 4 leukopenia ( $15 \%$ incidence), thrombocytopenia (10\%), and anaemia (5\%), as well as grade 3 esophagitis, occurred in $15 \%$ of the patients (30). The results of these studies were good compared to CRT using 5-FU and CDDP with a median survival time of $13-18$ months $(8,9,31)$. That suggested the combination of $\mathrm{S}-1$ and radiation could be a powerful regimen for controlling tumour progression with tolerable toxicity in patients with esophageal cancer.

We observed a high incidence of esophagitis compared to the previous studies on CRT with S-1 and platinum for 
non-age-selected patients with esophageal cancer, which could be explained by the differences of RT schedules. In the study by Iwase et al. (29), there was an interval of 2 weeks after 30 Gy RT so that the acute radiation injury of the oesophagus could be repaired partly. In the study of CRT with S-1 and nedaplatin, a boost dose was delivered to the primary tumour after doses of 41.4 Gy (30). In addition, studies have shown that age is an independent prognostic factor of radiation esophagitis, and the incidence of over grade 2 esophagitis was significantly increased in elderly patients (32). The toxicity profiles in our study were relatively similar to that reported in a phase I trial of preoperative CRT for squamous cell carcinoma of the oral cavity. The RD for S-1 was $65 \mathrm{mg} / \mathrm{m}^{2} 5$ days per week for 4 weeks when given concurrently with 40 Gy RT. The DLT in this study was mucositis, and the haematological toxicity was also mild (33).

In conclusion, the concurrent administration of S-1 and RT is well tolerated and promising in elderly patients with esophageal cancer. The RD of S-1 with concurrent RT for this protocol is $70 \mathrm{mg} / \mathrm{m}^{2}$ on days $1-14$ and $29-42$. We have already started a phase II study in multiple institutes to evaluate the efficacy and safety of this regimen.

\section{Acknowledgements}

Funding: This work was supported by the Foundation of Zhejiang Educational Committee (grant number Y201226285).

\section{Footnote}

Conflicts of Interest: The authors have no conflicts of interest to declare.

\section{References}

1. Ferlay J, Shin HR, Bray F, et al. Estimates of worldwide burden of cancer in 2008: GLOBOCAN 2008. Int J Cancer 2010;127:2893-917.

2. Trivers KF, Sabatino SA, Stewart SL. Trends in esophageal cancer incidence by histology, United States, 1998-2003. Int J Cancer 2008;123:1422-8.

3. Jemal A, Bray F, Center MM, et al. Global cancer statistics. CA Cancer J Clin 2011;61:69-90.

4. Wu M, Van't Veer P, Zhang ZF, et al. A large proportion of esophageal cancer cases and the incidence difference between regions are attributable to lifestyle risk factors in
China. Cancer Lett 2011;308:189-96.

5. Steyerberg EW, Neville B, Weeks JC, et al. Referral patterns, treatment choices, and outcomes in locoregional esophageal cancer: a population-based analysis of elderly patients. J Clin Oncol 2007;25:2389-96.

6. Tougeron D, Hamidou H, Scotté M, et al. Esophageal cancer in the elderly: an analysis of the factors associated with treatment decisions and outcomes. BMC Cancer 2010;10:510.

7. Cronin-Fenton DP, Sharp L, Carsin AE, et al. Patterns of care and effects on mortality for cancers of the oesophagus and gastric cardia: a population-based study. Eur J Cancer 2007;43:565-75.

8. Cooper JS, Guo MD, Herskovic A, et al. Chemoradiotherapy of locally advanced esophageal cancer: long-term follow-up of a prospective randomized trial (RTOG 85-01). Radiation Therapy Oncology Group. JAMA 1999;281:1623-7.

9. Herskovic A, Martz K, al-Sarraf M, et al. Combined chemotherapy and radiotherapy compared with radiotherapy alone in patients with cancer of the esophagus. N Engl J Med 1992;326:1593-8.

10. Tougeron D, Di Fiore F, Thureau S, et al. Safety and outcome of definitive chemoradiotherapy in elderly patients with oesophageal cancer. Br J Cancer 2008;99:1586-92.

11. Takeuchi S, Ohtsu A, Doi T, et al. A retrospective study of definitive chemoradiotherapy for elderly patients with esophageal cancer. Am J Clin Oncol 2007;30:607-11.

12. Semrau R, Herzog SL, Vallböhmer D, et al. Radiotherapy in elderly patients with inoperable esophageal cancer. Is there a benefit? Strahlenther Onkol 2012;188:226-32.

13. Wakui R, Yamashita H, Okuma K, et al. Esophageal cancer: definitive chemoradiotherapy for elderly patients. Dis Esophagus 2010;23:572-9.

14. Shirasaka T, Shimamato Y, Ohshimo H, et al. Development of a novel form of an oral 5-fluorouracil derivative (S-1) directed to the potentiation of the tumor selective cytotoxicity of 5 -fluorouracil by two biochemical modulators. Anticancer Drugs 1996;7:548-57.

15. Koizumi W, Kurihara M, Nakano S, et al. Phase II study of S-1, a novel oral derivative of 5 -fluorouracil, in advanced gastric cancer. For the S-1 Cooperative Gastric Cancer Study Group. Oncology 2000;58:191-7.

16. Koizumi W, Narahara H, Hara T, et al. S-1 plus cisplatin versus $\mathrm{S}-1$ alone for first-line treatment of advanced gastric cancer (SPIRITS trial): a phase III trial. Lancet Oncol 2008;9:215-21. 
17. Kawahara M, Furuse K, Segawa Y, et al. Phase II study of $\mathrm{S}-1$, a novel oral fluorouracil, in advanced non-small-cell lung cancer. Br J Cancer 2001;85:939-43.

18. Shiroyama T, Komuta K, Imamura F, et al. Phase II study of S-1 monotherapy in platinum-refractory, advanced nonsmall cell lung cancer. Lung Cancer 2011;74:85-8.

19. Van den Brande J, Schöffski P, Schellens JH, et al. EORTC Early Clinical Studies Group early phase II trial of S-1 in patients with advanced or metastatic colorectal cancer. Br J Cancer 2003;88:648-53.

20. van Groeningen CJ, Peters GJ, Schornagel JH, et al. Phase I clinical and pharmacokinetic study of oral S-1 in patients with advanced solid tumors. J Clin Oncol 2000;18:2772-9.

21. Yamada Y, Hamaguchi T, Goto M, et al. Plasma concentrations of 5-fluorouracil and F-beta-alanine following oral administration of S-1, a dihydropyrimidine dehydrogenase inhibitory fluoropyrimidine, as compared with protracted venous infusion of 5-fluorouracil. Br J Cancer 2003;89:816-20.

22. Takagi M, Sakata K, Someya M, et al. Gimeracil sensitizes cells to radiation via inhibition of homologous recombination. Radiother Oncol 2010;96:259-66.

23. Fukushima M, Sakamoto K, Sakata M, et al. Gimeracil, a component of S-1, may enhance the antitumor activity of X-ray irradiation in human cancer xenograft models in vivo. Oncol Rep 2010;24:1307-13.

24. Charlson ME, Pompei P, Ales KL, et al. A new method of classifying prognostic comorbidity in longitudinal studies: development and validation. J Chronic Dis 1987;40:373-83.

25. Therasse P, Arbuck SG, Eisenhauer EA, et al. New guidelines to evaluate the response to treatment in solid tumors. European Organization for Research and Treatment of Cancer, National Cancer Institute of the

Cite this article as: Ji Y, Qiu G, Sheng L, Sun X, Zheng Y, Chen M, Du X. A phase I dose escalation study of S-1 with concurrent radiotherapy in elderly patients with esophageal cancer. J Thorac Dis 2016;8(3):451-458. doi: 10.21037/ jtd.2016.02.70
United States, National Cancer Institute of Canada. J Natl Cancer Inst 2000;92:205-16.

26. Tahara M, Ohtsu A, Hironaka S, et al. Clinical impact of criteria for complete response (CR) of primary site to treatment of esophageal cancer. Jpn J Clin Oncol 2005;35:316-23.

27. Anderson SE, Minsky BD, Bains M, et al. Combined modality chemoradiation in elderly oesophageal cancer patients. Br J Cancer 2007;96:1823-7.

28. Servagi-Vernat S, Bosset M, Crehange G, et al. Feasibility of chemoradiotherapy for oesophageal cancer in elderly patients aged >or $=75$ years: a prospective, single-arm phase II study. Drugs Aging 2009;26:255-62.

29. Iwase H, Shimada M, Tsuzuki T, et al. Concurrent chemoradiotherapy with a novel fluoropyrimidine, S-1, and cisplatin for locally advanced esophageal cancer: longterm results of a phase II trial. Oncology 2013;84:342-9.

30. Tsuda T, Inaba H, Miyazaki A, et al. Prospective study of definitive chemoradiotherapy with $\mathrm{S}-1$ and nedaplatin in patients with stage II/III (non-T4) esophageal cancer. Esophagus 2011;8:45-51.

31. Minsky BD, Pajak TF, Ginsberg RJ, et al. INT 0123 (Radiation Therapy Oncology Group 94-05) phase III trial of combined-modality therapy for esophageal cancer: high-dose versus standard-dose radiation therapy. J Clin Oncol 2002;20:1167-74.

32. Ahn SJ, Kahn D, Zhou S, et al. Dosimetric and clinical predictors for radiation-induced esophageal injury. Int J Radiat Oncol Biol Phys 2005;61:335-47.

33. Harada H, Omura K. Preoperative concurrent chemotherapy with S-1 and radiotherapy for locally advanced squamous cell carcinoma of the oral cavity: phase I trial. J Exp Clin Cancer Res 2010;29:33. 